\title{
Safety of the long-term application of QuikClot Combat Gauze, ChitoGauze PRO and Celox Gauze in a femoral artery injury model in swine - a preliminary study
}

\author{
I. Otrocka-Domagała ${ }^{1}$, P. Jastrzębski ${ }^{2}$, Z. Adamiak ${ }^{3}$, K. Paździor-Czapula ${ }^{1}$, \\ M. Gesek ${ }^{1}$, M. Mikiewicz ${ }^{1}$, T. Rotkiewicz ${ }^{1}$ \\ ${ }^{1}$ Department of Pathological Anatomy, Faculty of Veterinary Medicine, \\ University of Warmia and Mazury in Olsztyn, Oczapowskiego 13, 10-719 Olsztyn, Poland \\ ${ }^{2}$ Security Fundamentals Unit, Police Academy, Piłsudskiego 111, 12-100 Szczytno, Poland \\ ${ }^{3}$ Department of Surgery and Radiology, Faculty of Veterinary Medicine, \\ University of Warmia and Mazury, Oczapowskiego 14, 10-719 Olsztyn, Poland
}

\begin{abstract}
The purpose of this study was to examine the safety of the long-term application of QuikClot Combat Gauze, ChitoGauze PRO and Celox Gauze using a swine model. The study was conducted on nine pigs weighing approximately $30 \mathrm{~kg}$, which were randomly divided into three groups. Under deep anesthesia, the pigs underwent complete transverse cutting of the femoral artery in the groin region. Hemostatic dressings were left in the wound for 24 hours. The animals were euthanized 24 hours after dressing application. In each group, macroscopic and microscopic severe changes and shock symptoms were observed in the lungs, liver, kidneys and heart. Fibrino-gaseous embolic material was found in the pulmonary artery of each group and in the lung vessels of the animals from the ChitoGauze PRO and Celox Gauze groups. In conclusion, the long-term application of the evaluated hemostatic dressings has the risk of coagulopathy and reaching the progressive stage of shock. The residues from the hemostatic dressings can ingress into the systemic circulation, thereby increasing the risk of embolus formation. Because of these harmful effects, the evaluated hemostatic dressings are not appropriate for long-term use. Future studies are needed on the consequences of the long-term application of these hemostatic agents.
\end{abstract}

Key words: hemostatic dressings, kaolin, chitosan, shock, thromboemboli, swine model 


\section{Introduction}

Arterial and venal injuries can reduce the circulating blood volume in a very short time and can lead to hypotension as well as cellular and tissue hypoperfusion. Hypotension and hypoperfusion cause peripheral vasoconstriction, movement of the interstitial fluid into the plasma and an increased heart rate. These compensatory mechanisms increase the vascular pressure to achieve sufficient perfusion for the heart, brain and kidneys; therefore, shock is stabilized in the nonprogressive stage. The compensatory mechanisms can occasionally return the system to homeostasis, but if the hemorrhage is severe or long-lasting, the shock develops into the progressive stage. In this stage, there is blood pooling and stagnation, which contribute to embolization, hypoperfusion, progressive tissue damage and necrosis. In addition to being caused by insufficient oxygenation, tissue damage results from the release of the mediators that are specific for the progressive stage of shock, such as histamine, kinins, platelet-activating factor (PAF), complement fragments, and a wide variety of cytokines. These mediators are responsible for the systemic activation of complement, coagulation, fibrynolysis, inappropriate systemic inflammation and the kinin system pathway. The prolonged progressive stage of shock results in the multiple organ dysfunction, particularly in the lungs, liver, intestines, kidneys, and the heart, and it leads to the irreversible stage of shock and death. Finally, the shock is manifested as disseminated intravascular coagulation (DIC), which is part of severe homeostasis dysfunction (Gutierrez et al. 2004, Mosier 2012).

Currently, there are research efforts to identify efficient, fast-acting hemostatic materials that could be used in extensive cut injuries, lacerations and gunshot wounds, to prevent shock and its consequences, which is highly important for military combat units (King 2011). Military combat units use many different agents as hemostatic dressings, including zeolite, chitosan and kaolin (Clay et al. 2010, De Castro et al. 2011). Zeolite is a very effective water-absorbing aluminosilicate that acts via a non-chemical reaction. At the site of bleeding, zeolite effectively concentrates platelets and clotting factors, but it also releases $\mathrm{Ca}^{2+}$ into the blood, which accelerates the intrinsic pathway of blood coagulation and shortens the clot formation time ( $\mathrm{Li}$ et al. 2013). However, upon contact with blood, zeolite causes an exothermic reaction, which damages the surrounding tissue (Wright et al. 2004, Li et al. 2013). Therefore, due to the high risk of tissue injury and microemboli formation, zeolite hemostatic dressings were removed from the United States military inventory (Gegel et al. 2012). Kaolin is another hemostatic agent that promotes clot formation by activating factors XII and factor XI, which form the intrinsic clotting pathway; its application ends with the formation of a fibrin clot (Johnson et al. 2014). Interestingly, because hemostatic agents containing kaolin do not cause an exothermic effect, they cause less tissue injury and thromboemboli formation. Chitosan and its salts bind with platelets and red blood cells in an electrostatic reaction to form a gel-like clot that seals a bleeding vessel. In this way, chitosan acts independent of the classical coagulation pathway. Chitosan does not cause an exothermic reaction, and it has antibacterial properties (Millner et al. 2010, De Castro et al. 2011).

The usefulness of the hemostatic dressings in patients with massive hemorrhage is determined by the rapidity of the chirurgical intervention in the area of vascular injury (King 2011). Long-term application of hemostatic dressings, which is occasionally necessary during military transportation, can cause side effects, including slow progressing shock, intravascular coagulation, embolization and damage to many internal organs. Additionally, hemostatic dressings have local tissue irritating effects that can be harmful (Adamiak et al. 2014).

The aim of this study was to evaluate the influence of a 24-hour application of the kaolin-containing QuikClot Combat Gauze, chitosan-containing ChitoGauze PRO, and chitosan-containing Celox Gauze on internal organs during femoral artery injury in a swine model, particularly with respect to shock prevention.

\section{Materials and Methods}

The local ethics committee approved the experiments described in this report (No. 84/2012), which were conducted in accordance with the provisions of the Guide for the Care and Use of Laboratory Animals. The study was performed on 9 Polish Large White female pigs with a body weight of $30 \mathrm{~kg}$; the pigs were equally divided into three groups. QuikClot Combat Gauze (Z-Medica Corporation, Wallingford, CT, USA) containing kaolin was applied in the first group. ChitoGauze PRO (HemCon Medical Technologies, Inc., Portland, OR, USA), coated with chitosan, was used in the second group. Celox Gauze (Medtrade Products Ltd., Crewe, UK), a high-density gauze impregnated by chitosan granules, was applied in the third group. The animals were premedicated with azaperone (Stresnil, Janssen Pharmaceutica N.V., Beerse, Belgium), administered intramuscularly at $2.0 \mathrm{mg} / \mathrm{kg}$; atropine (Atropinum Sulfuricum, Polfa S.A, Warsaw, Poland), administered intramuscularly 
Table 1. Pathological changes in the internal organs of pigs 24 hours after applying a hemostatic dressing. The number of pigs from the QuikClot Combat Gauze, ChitoGauze PRO and Celox Gauze groups showing particular pathological changes.

\begin{tabular}{|c|c|c|c|}
\hline Pathological changes & $\begin{array}{l}\text { QuikClot Combat Gauze } \\
\qquad \mathrm{n}=3\end{array}$ & $\begin{array}{c}\text { ChitoGauze PRO } \\
n=3\end{array}$ & $\begin{array}{c}\text { Celox Gauze } \\
n=3\end{array}$ \\
\hline \multicolumn{4}{|l|}{ Lungs } \\
\hline focal inflammation & 2 & 2 & 2 \\
\hline focal acute alveolar emphysema & 3 & 2 & 1 \\
\hline focal atelectasis & 1 & 1 & 1 \\
\hline focal hemorrhagic infarctions & 1 & 1 & 1 \\
\hline subpleural petechiae & 1 & 2 & 2 \\
\hline hyperemia with edema & 2 & 3 & 3 \\
\hline \multicolumn{4}{|l|}{ Pulmonary artery } \\
\hline fibrino-gaseous embolus & 2 & 3 & 3 \\
\hline \multicolumn{4}{|l|}{ Heart } \\
\hline subepicardiac and myocardiac petechiae & 3 & 2 & 1 \\
\hline hyperemia & 2 & 2 & 1 \\
\hline myocardial degeneration & 3 & 2 & 1 \\
\hline \multicolumn{4}{|l|}{ Kidneys } \\
\hline shock kidney & 1 & 0 & 2 \\
\hline hyperemia & 3 & 2 & 1 \\
\hline \multicolumn{4}{|l|}{ Liver } \\
\hline hyperemia & 2 & 1 & 1 \\
\hline multifocal purulent inflammation & 1 & 0 & 0 \\
\hline \multicolumn{4}{|l|}{ Peritoneal cavity } \\
\hline abscesses in the omentum and area of the urinary bladder & 1 & 0 & 0 \\
\hline
\end{tabular}

Table 2. Histopathological changes in the lungs, kidneys and livers of pigs 24 hours after hemostatic dressing application. The number of pigs from QuikClot Combat Gauze, ChitoGauze PRO and Celox Gauze groups showing particular pathological changes.

Histopathological changes

QuikClot Combat Gauze ChitoGauze PRO Celox Gauze
$\mathrm{n}=3$
$\mathrm{n}=3$
$\mathrm{n}=3$

\begin{tabular}{|c|c|c|c|}
\hline Lungs & & & \\
\hline acute alveolar emphysema & 3 & 2 & 1 \\
\hline non-purulent peribronchitis & 2 & 2 & 1 \\
\hline non-purulent interstitial pneumonia & 2 & 2 & 1 \\
\hline catarrhal pneumonia & 1 & 1 & 1 \\
\hline focal purulent pneumonia & 1 & 1 & 1 \\
\hline hyperemia, edema & 2 & 3 & 3 \\
\hline focal/massive atelectasis & 1 & 1 & 1 \\
\hline disseminated intravascular coagulation & 2 & 2 & 2 \\
\hline fibrino-gaseous emboli in lung vessels & 0 & 2 & 1 \\
\hline focal hemorrhagic infarctions & 1 & 1 & 1 \\
\hline Kidneys & & & \\
\hline acute cortical necrosis & 1 & 0 & 1 \\
\hline degeneration of the tubular epithelium & 2 & 2 & 2 \\
\hline hyperemia of the arcuate vessels, small extravasations & 1 & 0 & 2 \\
\hline hyperemia & 3 & 2 & 1 \\
\hline Liver & & & \\
\hline degeneration of the hepatocytes & 1 & 1 & 1 \\
\hline hyperemia & 2 & 1 & 1 \\
\hline lymphocytic infiltrates in interstitial tissue and portal fields & 1 & 0 & 0 \\
\hline multifocal purulent hepatitis & 1 & 0 & 0 \\
\hline
\end{tabular}


at $0.05 \mathrm{mg} / \mathrm{kg}$; and butorphanol (Butomidor, Richter Pharma AG, Wels, Austria), administered intravenously at $0.2 \mathrm{mg} / \mathrm{kg}$. General anesthesia was induced with ketamine (Bioketan, Vetoquinol S.A., Lure, France), administered intramuscularly at $8 \mathrm{mg} / \mathrm{kg}$, and maintained with thiopental natricum (Pentothal, Abbott Laboratories Poland, Warsaw, Poland), administered intravenously at $10 \mathrm{mg} / \mathrm{kg}$. Hemostatic agents were tested using a lethal femoral artery injury model. A transverse incision was made across the entire femoral artery. After 45 seconds of hemorrhage, the animals were randomized for treatment with one of the three hemostatic dressings (three pigs for each dressing). The selected hemostatic dressing was applied to the wound and manually compressed for 3 to 5 minutes. The wound, with a hemostatic dressing inside, was closed with a continuous suture. The pigs were euthanized 24 hours after application of the hemostatic agents via intravenous injection of pentobarbital (Morbital, Biowet, Pulawy, Poland). After euthanasia, necropsy was performed, and the internal organs (lungs, liver and kidneys) were collected for histopathology. The collected specimens were fixed in $10 \%$ neutral buffered formalin, embedded in paraffin and routinely stained with hematoxylin and eosin (HE). The effect of staining was evaluated in preparations that were scanned with a digital histology slide scanner (3DHISTECH, Hungary).

\section{Results}

All hemostatic dressings successfully stopped bleeding in all of the pigs that survived 24 hours after femoral artery injury. There was no dehiscence or dressing prolapse. Necropsy revealed morphological changes in the lungs, kidneys, liver and heart, indicating shock (focal atelectasis; edema and hyperemia in the lungs; hyperemia of the arcuate vessels and acute cortical necrosis in the kidneys; degeneration and hyperemia of the liver and cardiac muscle; and subpleural, subepicardial and myocardial ecchymoses). In all of the evaluated groups, fibrino-gaseous emboli were confirmed in the pulmonary artery. In the animals treated with ChitoGauze PRO, the embolic material was larger and more distinct than that observed in the other groups. In the QuikClot Combat Gauze and Celox Gauze groups, the embolic material was smaller and had the appearance of more gas. Detailed morphological changes in every evaluated organ in each evaluated group are given in table 1 .

The microscopic examination of the collected lungs, liver and kidneys confirmed the presence of the lesions that had been observed macroscopically. Table
2 summarizes the microscopic lesions of the internal organs examined. Furthermore, the microscopic examination of the embolic material found in the pulmonary artery of the investigated pigs confirmed its fibrino-gaseous structure (Fig. 1a). Additionally, microscopic examination of the lungs revealed the presence of embolic material in the lung vessels that was similar in two animals from the ChitoGauze PRO group (Fig. 1) and one animal from the Celox Gauze group. The pigs from each group had disseminated intravascular coagulation in their lungs (Fig. 2).

\section{Discussion}

Uncontrolled extensive hemorrhage can cause circulatory disturbances and death in a short time. Therefore, bleeding from trauma often increases the morbidity and mortality in both civilians and soldiers. Early control of the hemorrhage is crucial for both survival and optimal recovery, especially when an individual must be transported over a long distance to a medical treatment facility. Therefore, many hemostatic agents have been investigated in multiple animal studies to create the most effective hemostatic dressing for controlling hemorrhage. Many of these agents, particularly those made of zeolite or used in the form of granular and fine powders, have been removed from use because of potential complications, especially thermal tissue injury at the site of trauma or microemboli formation, which are common adverse effects (Kheirabadi et al. 2010, Gegel et al. 2012). The newest agents, which consist of gauze dressings impregnated with kaolin and chitosan, are more effective and simple to use, and have a low risk of tissue injury and thromboemboli formation (Kheirabadi et al. 2010, Johnson et al. 2014). The majority of studies on the effectiveness of hemostatic agents using animal models have examined the influence of a short-term dressing application that was no longer than a few hours (Arnaud et al. 2009, Kheirabadi et al. 2010, Littlejohn et al. 2011, Gegel et al. 2012). There are no previously published studies on the consequences of the long-term application of hemostatic dressing, which is occasionally necessary on the battlefield, especially when the individual must be transported hundreds of kilometers to a medical facility.

The results of this study indicate that the evaluated hemostatic dressings have a risk of thromboemboli formation and damage to the crucial organs, such as the heart, lungs, liver and kidneys, with long-term application. The thrombosis observed in the lung vessels in all of the evaluated groups is the evidence of DIC and trauma-induced coagulopathy, which leads to the progressive phase of shock (Gando and Otomo 


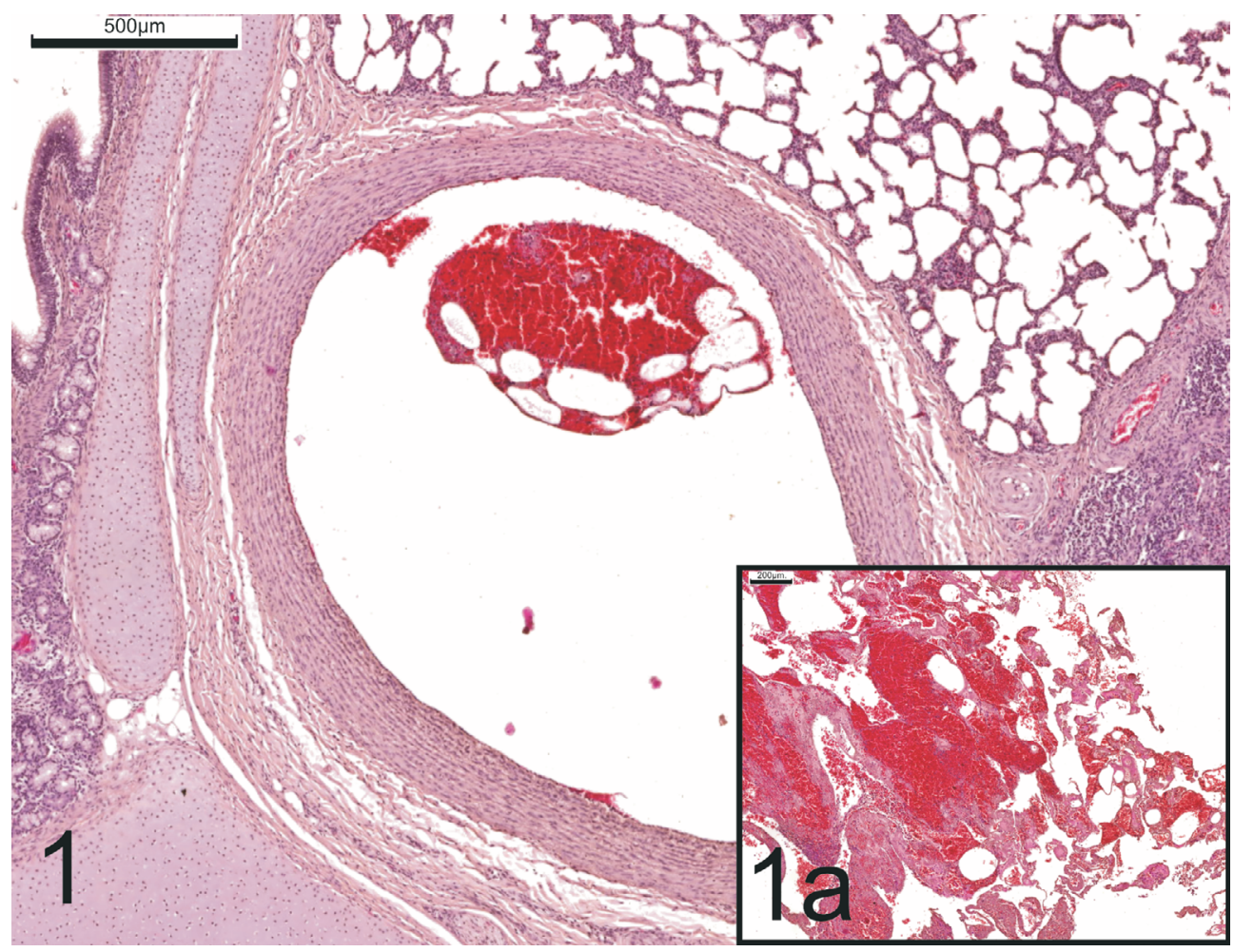

Fig. 1. Section of a lung tissue sample collected from the pig of ChitoGauze PRO group, stained with hematoxylin and eosin. Fibrino-gaseous embolic material found in a lung vessel. Inset (1A): The same fibrino-gaseous embolic material found in the pulmonary artery. Scale bar $\approx 500 \mu \mathrm{m}$.

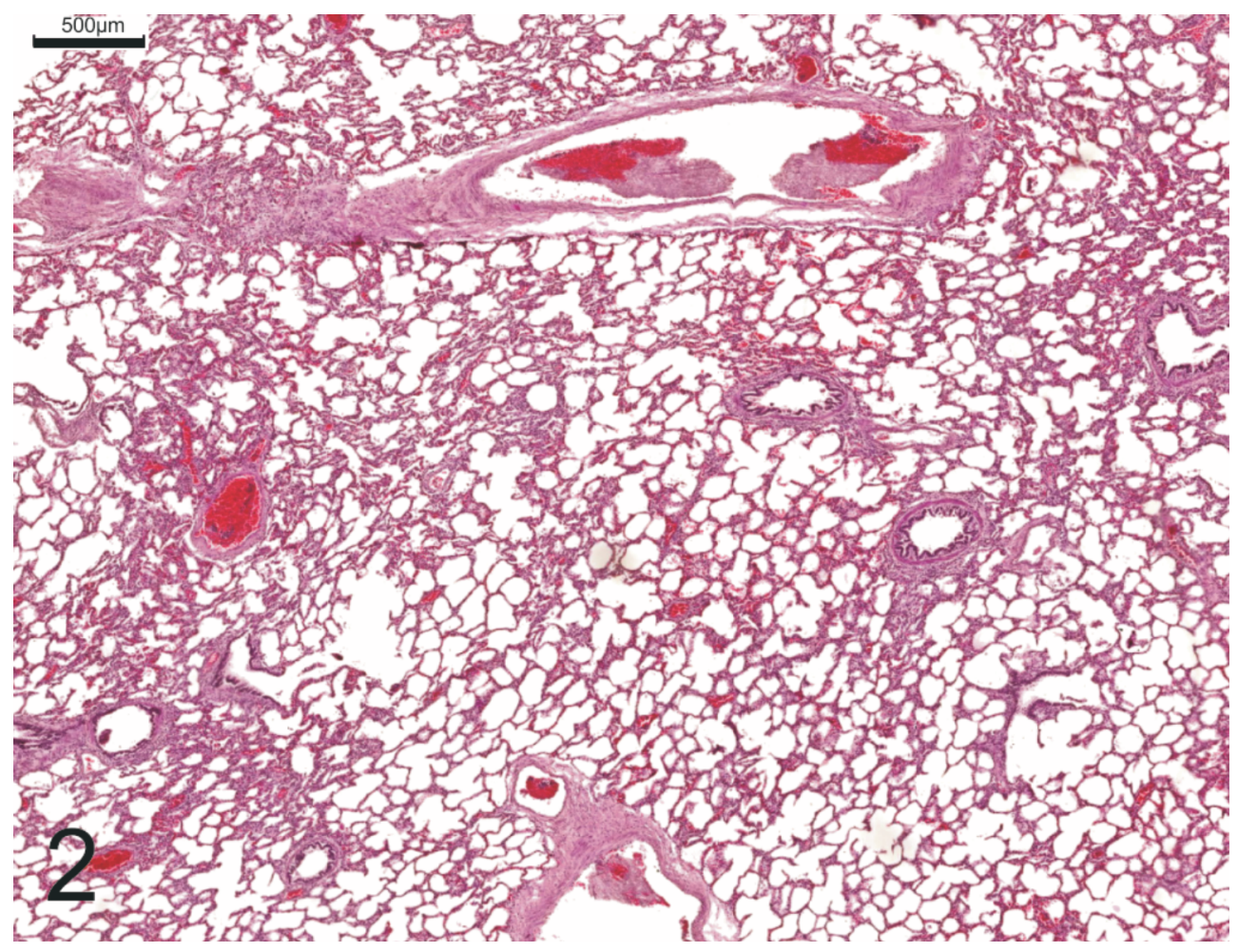

Fig. 2. Section of a lung tissue sample collected from the pig of QuikClot Gauze group, stained with hematoxylin and eosin Disseminated intravascular coagulation observed in the vessels. Scale bar $\approx 500 \mu \mathrm{m}$. 
2015). These results correspond to an increased level of the D-dimer concentration, as observed during our experiment, that was previously reported by Jastrzębski et al. (2014). Therefore, the long-term application of the hemostatic dressings used in this experiment do not prevent the development of the progressive stage of shock. Leandoer and Bergentz (1970) obtained similar results in a dog model of hemorrhagic shock. In response to antifibrinolytic therapy using tranexamic acid, they observed microvascular thrombosis and thromboemboli formation in the portal vein and lung vessels. Microemboli formation was also observed in studies on the effectiveness of zeolite dressings; however, studies on newer generation agents treated with kaolin or chitosan have not revealed their serious side effects (Pusateri et al. 2006, Clay et al. 2010, Gerlach et al. 2010, Kheirabadi et al. 2010, Littlejohn et al. 2011). In the present study, the coagulopathy and circulatory distress resulted in damage to the lungs, liver, kidneys and heart, which is a sign of the prolonged progressive stage of shock. These symptoms have never been observed in studies on the short-term application of different hemostatic dressings treated with kaolin or chitosan (Clay et al. 2010, Kheirabadi et al. 2010, Littlejohn et al. 2011).

Interestingly, the application of hemostatic dressings for 24 hours caused a slow release of the material fragments into the bloodstream. As a result, fibrino-gaseous embolic material was observed in the pulmonary arteries of the pigs in each evaluated group. Additionally, the same embolic material was observed in the lung vessels of the animals in the ChitoGauze PRO and Celox Gauze groups. This observation has never been reported in studies on the effectiveness of chitosan or kaolin impregnated hemostatic agents. However, Murphy et al. (1993), in a study on endothelial cell cultures, confirmed the potential toxic effect of kaolin on cells, but this effect was less dramatic than that observed for other hemostatic agents. Kheirabadi et al. (2010) observed severe endothelial injury and transmural damage with microscopic residues of the dressing in the systemic circulation, which was followed by distal thromboembolism in the carotid arteries, jugular vein and small lung vessels, in a study on the safety of different hemostatic agents at 2 hours after their application in pigs treated with clay mineral smectite (WoundStat). The authors did not observe thrombosis in the animals that were treated with kaolin-coated dressings. Similarly, Gerlach et al. (2010) observed endothelial injury and perivascular fibrogranulomatous inflammation in the spleen, and histiocytes containing WoundStat particles in the regional lymph node. They concluded that the pres- ence of dressing remnants in these organs is caused by macrophage phagocytic activity instead of particle embolization.

Local cytotoxicity of old generation hemostatic agents treated with smectite or zeolite has been observed in many studies (Wright et al. 2004, Gerlach et al. 2010, Kheirabadi et al. 2010, Li et al. 2013). However, there are no previous reports on the distant toxic effect of the hemostatic dressing. Therefore, the degenerative changes observed in the kidneys, liver and heart in the present study seem to be the effect of the progressive stage of shock rather than the result of the direct injury, secondary to dressing particles flowing through the blood. In contrast, the inflammatory foci observed in the pigs that were treated with kaolin and chitosan dressings could indicate that there is multiple organ dysfunction syndrome or penetration of the particles into the lungs via the bloodstream or macrophages. Unfortunately, we did not use polarized light to evaluate the dressing remnants in the organs examined. Abscesses in the peritoneal cavity and multifocal purulent hepatitis were confirmed in one animal from the QuikClot Combat Gauze group and should be regarded as incidental finding rather than the result of the hemostatic dressing application.

The form of a hemostatic agent impacts the removability of dressing residues from the wound as well as the potentially harmful effects (Arnaud et al. 2009, Clay et al. 2010, Kheirabadi et al. 2010). Herein, we did not observe any differences in the macroscopic or microscopic changes in the internal organs among the groups examined. Furthermore, circulatory disturbances, DIC, degenerative changes, pneumonia and thromboembolic material in the pulmonary artery and lung vessels were observed in all the groups independent of the type of the hemostatic agent. An explanation for the results obtained in our study could be the harmful systemic effect of the long-term application of QuikClot Combat Gauze, ChitoGauze PRO and CeloxGauze. The evaluated hemostatic agents were very effective in hemorrhage control, but they did not prevent the development of the progressive stage of shock. Moreover, their long-term application caused muscle, vessel and soft tissue damage at the site of use, which has been shown in our previous study (Adamiak et al. 2014). This could explain the slow progression of shock and entry of the dressing particles into the bloodstream with subsequent embolus formation.

The use of a hemostatic agent is one of the easiest and the most effective methods for treating extensive bleeding and preventing complications and death. The short-time use of new generation dressings is safe and does not cause serious side effects. However, their long-term application carries a risk of co- 
agulopathy and the development of the progressive stage of shock. Furthermore, with long-term use of hemostatic agents in dressings, residues from the dressing can ingress into the systemic circulation, thereby increasing the risk of embolus formation. Due to these harmful effects, QuikClot Combat Gauze, ChitoGauze PRO and CeloxGauze are not appropriate for long-term use. Our study represents a preliminary effort and the results obtained require confirmation with a larger study population.

\section{References}

Adamiak Z, Jastrzębski P, Pomianowski A, Otrocka-Domagała I, Holak P, Zhalniarovich Y, Przyborowska P, Głodek J (2014) Effect of 24 hour application of three hemostatic dressings to porcine thigh muscles. Pol J Vet Sci 17: 519-521.

Arnaud F, Teranishi K, Tomor T, Carr W, McCarron $R$ (2009) Comparison of 10 hemostatic dressings in a groin puncture model in swine. $\mathrm{J}$ Vasc Surg. 50: 632-639.

Clay JG, Grayson JK, Zierold D (2010) Comparative testing of new hemostatic agents in a swine model of extremity arterial and venous hemorrhage. Mil Med 175: 280-284.

De Castro GP, Dowling MB, Kilbourne M, Keledjian K, Driscoll IR, Raghavan SR, Hess JR, Scalea TM, Bochicchio GV (2011) Determination of efficacy of novel modified chitosan sponge dressing in a lethal arterial injury model in swine. J Trauma 72: 899-907.

Gando S, Otomo Y (2015) Local hemostasis, immunothrombosis, and systemic disseminated intravascular coagulation in trauma and traumatic shock. Crit Care 19: 72.

Gegel B, Burgert J, Gasko J, Campbell C, Martens M, Keck J, Reynolds H, Loughren M, Johnson D (2012) The effects of QuikClot Combat Gauze and movement on hemorrhage control in a porcine model. Mil Med 177: 1543-1547.

Gerlach T, Grayson JK, Pichakron KO, Sena MJ, DeMartini SD, Clark BZ, Estep JS, Zierold D (2010) Preliminary study of the effects of smectite granules (WoundStat) on vascular repair and wound healing in a swine survival model. J Trauma 69: 1203-1209.

Gutierrez G, Reines HD, Wulf-Gutierrez ME (2004) Clinical review: hemorrhagic shock. Crit Care 8: 373-381.

Jastrzębski P, Adamiak Z, Pomianowski A, Krystkiewicz W, Holak P, Sawicki S, Przyborowska P, Zhalniarovich Y,
Gudzbeler G (2014) Response of the coagulation system after application of hemostatic dressings in an animal model. Pol J Vet Sci 17: 725-727.

Johnson D, Bates S, Nukalo S, Staub A, Hines A, Leishman T, Michel J, Sikes D, Gegel B, Burgert J (2014) The effects of QuikClot Combat Gauze on hemorrhage control in the presence of hemodilution and hypothermia. Ann Med Surg (Lond) 3: 21-25.

Kheirabadi BS, Mace JE, Terrazas IB, Fedyk CG, Estep JS, Dubick MA, Blackbourne LH (2010) Safety evaluation of new hemostatic agents, smectite granules, and kaolin-coated gauze in a vascular injury wound model in swine. J Trauma 68: 269-278.

King DR (2011) Thirty consecutive uses of a hemostatic bandage at a US Army combat support hospital and forward surgical team in Operation Iraqi Freedom. J Trauma 71: 1775-1778.

Leandoer L, Bergentz SE (1970) Haemorrhagic shock in the dog. The formation of thromboemboli during antifibrinolytic therapy. Eur Surg Res 2: 341-347.

Li J, Cao W, LvXX, Jiang L, Li YJ, Li WZ, Chen SZ, Li XY (2013) Zeolite-based hemostat QuikClot releases calcium into blood and promotes blood coagulation in vitro. Acta Pharmacol Sin 34: 367-372.

Littlejohn LF, Devlin JJ, Kircher SS, Lueken R, Melia MR, Johnson AS (2011) Comparison of Celox-A, ChitoFlex, WoundStat, and combat gauze hemostatic agents versus standard gauze dressing in control of hemorrhage in a swine model of penetrating trauma. Acad Emerg Med 18: 340-350.

Millner R, Lockhart AS, Marr R (2010) Chitosan arrests bleeding in major hepatic injuries with clotting dysfunction: an in vivo experimental study in a model of hepatic injury in the presence of moderate systemic heparinisation. Ann R Coll Surg Engl 92: 559-561.

Mosier DA (2012) Vascular disorders and thrombosis. In: Zachary JF, McGavin MD (eds) Pathologic basis of veterinary disease, 5th ed. Mosby Elsevier, St Louis, Missouri, pp 60-86.

Murphy EJ, Roberts E, Horrocks LA (1993) Aluminum silicate toxicity in cell cultures. Neuroscience 55: 597-605.

Pusateri AE, Holcomb JB, Kheirabadi BS, Alam HB, Wade CE, Ryan KL (2006) Making sense of the preclinical literature on advanced hemostatic products. J Trauma 60: 674-682.

Wright JK, Kalns J, Wolf EA, Traweek F, Schwarz S, Loeffler CK, Snyder W, Yantis LD Jr, Eggers J (2004) Thermal injury resulting from application of a granular mineral hemostatic agent. J Trauma 57: 224-230. 